\title{
The Crucial Role of Elite Athletes and Expert Coaches With Academic Profiles in Developing Sound Sport Science
}

Sport science and medicine are highly specialized fields, where many scientific disciplines can contribute to improving athletes' health and performance. In that regard, having athletes, coaches, and scientists working side by side on scientific research projects can significantly improve the quality of the science. I can illustrate this by my own modest experience as a sport scientist and former elite athlete. Indeed, in many scientific articles that I have handled as an author or as International Journal of Sports Physiology and Performance (IJSPP) associate editor, I have noticed how deep "sportdiscipline knowledge" greatly improved the quality of publications.

I hope that the recent call to reviewers ${ }^{1}$ in which we reminded all scientists about their roles in keeping science running had an impact. Indeed, without committed reviewers, the peer-review process would stop, and so would science. In this context, in the area of sport performance, I would like to highlight the fact that to obtain an optimal outcome of a sound and impactful paper, we need domain (sport) specialists' input at 2 important steps: (1) study conception and article writing and (2) the manuscript peer-review process.

To illustrate this point, I would highlight 2 recent articles, both being from the field of elite karate. In the first paper, ${ }^{2}$ I had the chance to work with 3 academics, 2 of whom were combat-sport specialists, with the leading author being an ex-world-class elite karateka. Without their input, the research would have been limited to practitioners in the field. Specifically, my biases and background would have pushed the study toward the viewpoint of an exercise physiologist with a different sport background. Most likely, the study would have been substantially different with a focus on classical physiological variables and concepts of internal and external load. The expert practitioners' involvement instead took us down a previously unexplored but ultimately fruitful line of exploring what happens before and after the "decisive moment" in karate combat - the moment from which one of the 2 opponents uninterruptedly dominates the other until the end of the fight, in simple terms, "how the fight was won." I like this paper not only because it clearly describes technical actions that lead to the decisive moment, and therefore, winning a karate combat, but also, and more important, because it could inspire other sport-scientific experts on this novel way of analyzing sport performance with regard to a crucial moment, when "victory has been definitely set." Without the domain specialists, this project would have had much less real-world impact.

The second example is from the peer-review process of another original article on karate that I coauthored. ${ }^{3}$ One of the anonymous reviewers of this paper was clearly an expert in karate and greatly improved the final product. All the very detailed and constructive comments made on the manuscript clearly showcased the reviewer's high level of expertise in this sport. As authors, we could not have been happier with this review process from such a rigorous, exigent, and competent reviewer. Among other things, the reviewer helped us better translate numbers in real sport actions to provide clear practical applications. Ultimately, the addition of this reviewer's domain knowledge coupled with clear scientific knowledge greatly improved this paper for the readers. We believe that the outcome of the review process clearly brought relevant data/knowledge on the technical and tactical discriminatory factors between winners and defeated elite karate athletes, expressed in a much clearer way, thanks to both sport and scientific expertise of the reviewers.

Reflecting on these specific papers, we suspect that their impact may go beyond karate and to inspire other sport scientists to investigate their sports regarding these crucial performance determinants. After all, isn't winning or losing a central concern of all coaches and sport scientists?

More broadly, these illustrations confirm a trend that I have noticed as associate editor while managing manuscript-review processes for IJSPP. Some reviewers are deep experts in their sports, and their influence undoubtedly enhances this quality and depth; on this much we can agree, as science always needs these attributes.

Therefore, I call on academics who are sport specialists to maintain their efforts in improving sport science in their fields. We need these people, not only as authors of manuscripts, but also, and perhaps even more important, as reviewers. And again, I implore us, as a group, to work toward encouraging their participation and removing whatever barriers are currently in place making it difficult for them to be involved in the review process. Perhaps a fault lies in the actual situation, giving too much credit to manuscript authors, and surely not enough recognition to reviewers, who are indeed the "real unsung heroes in science."

\section{Karim Chamari, Associate Editor, IJSPP}

\section{References}

1. Chamari K, Beneke R. The game is fair play: a kind reminder to scientists about their role in keeping science running. Int J Sports Physiol Perform. 2017; 12( 9): 1125- 1126. PubMed ID: 29214916 doi:10.1123/ijspp.2017-0615

2. Tabben M, Miarka B, Chamari K, Beneke R. Decisive moment: a metric to determine success in elite karate bouts. Int J Sports Physiol Perform. 2018; 13( 8): 1000- 1004. PubMed ID: 29345545 doi:10. 1123/ijspp.2017-0526

3. Tabben M, Conte D, Haddad M, Chamari K. Technical and tactical discriminatory factors between winners and defeated elite karate athletes. Int J Sports Physiol Perform. 2018; 9( 1): 1- 19. PubMed ID: 30300069 doi:10.1123/ijspp.2018-0478

4. Leithäuser RM. The unsung heroes in science. Int J Sports Physiol Perform. 2014; 9( 4): 597. PubMed ID: 24954848 doi:10.1123/ijspp. 2014-0143 